\title{
A SYMBOLIC CALCULUS FOR ANALYTIC CARLEMAN CLASSES
}

\author{
JAMIL A. SIDDIQI AND MOSTEFA IDER
}

\begin{abstract}
Let $\mathscr{C}_{M}\left(I_{\alpha}\right)$ be the analytic Carleman class of $\mathscr{C}^{\infty}$-functions $f$ defined in a sector $I_{\alpha}=\{z \in \mathbf{C}:|\arg z| \leqslant \alpha \pi / 2\} \cup\{0\}(0 \leqslant \alpha \leqslant 1)$ and analytic in its interior such that $\left\|f^{(n)}\right\|_{\infty} \leqslant C \lambda^{n} M_{n}(n \geqslant 0), C=C(f), \lambda=\lambda(f)$. In this paper, we give necessary and sufficient conditions in order that $\mathscr{C}_{M}\left(I_{\alpha}\right)$ be inverse-closed. As a corollary, we obtain a characterization of $\mathscr{C}_{M}\left(\mathbf{R}_{+}\right)$as an inverse-closed algebra, thus establishing the converse of a theorem of Malliavin [4] for the half-line.
\end{abstract}

1. Given a sequence $M=\left\{M_{n}\right\}$ of positive numbers, we say that the sequence $\left\{A_{n}\right\}$, where $A_{n}=\left(M_{n} / n !\right)^{1 / n}$, is almost increasing if there exists a positive constant $K$ such that for all $m$ and $n$ with $n \leqslant m, A_{n} \leqslant K A_{m}$. P. Malliavin [4] proved that if $M$ is log-convex and the associated sequence $A=\left\{A_{n}\right\}$ is almost increasing, then the algebra $\mathscr{C}_{M}(I)$ of $\mathscr{C}^{\infty}$-functions on a linear interval $I$ with

$$
\left\|f^{(n)}\right\|_{\infty} \leqslant C \lambda^{n} M_{n} \quad(n \geqslant 0), \quad C=C(f), \quad \lambda=\lambda(f),
$$

is inverse closed, i.e., if $f \in \mathscr{C}_{M}(I)$ and $f(x) \neq 0$ for all $x \in I$, then $f^{-1} \in \mathscr{C}_{M}(I)$. The problem whether the converse is true, viz., if $\mathscr{C}_{M}(I)$ is inverse closed then the sequence $A$ is almost increasing, was taken up by W. Rudin [6] who proved that it is so if $\mathscr{C}_{M}(R)$ is a non-quasi-analytic algebra of $2 \pi$-periodic functions. Subsequently J. Boman and L. Hörmander [1] proved the same result for arbitrary classes $\mathscr{C}_{M}(\mathbf{R})$ by a long and ingenious method. The problem whether the converse of Malliavin's theorem is true for classes $\mathscr{C}_{M}(I)$, where $I$ is a half-line or a finite interval, remains unsolved. Recently J. Bruna [2] studied the related problem for Beurling classes.

In this paper we give a necessary and sufficient condition in order that the algebra $\mathscr{C}_{M}\left(I_{\alpha}\right)$ of $\mathscr{C}^{\infty}$-functions $f$ in a sector $I_{\alpha}=\{z \in \mathbf{C}:|\arg z| \leqslant \alpha \pi / 2\} \cup\{0\}(0 \leqslant \alpha$ $\leqslant 1)$ and analytic in its interior such that $\left\|f^{(n)}\right\|_{\infty} \leqslant C \lambda^{n} M_{n}, C=C(f), \lambda=\lambda(f)$, be inverse-closed. As a corollary we obtain a characterization of the inverse-closed algebra $\mathscr{C}_{M}\left(\mathbf{R}_{+}\right)$, thus establishing the converse of Malliavin's theorem for the half-line.

2. The analytic Carleman classes $\mathscr{C}_{M}\left(I_{\alpha}\right)$ have been extensively studied by several authors, notably by B. I. Korenbljum [3] who gave conditions for the nontriviality and the quasi-analyticity of these classes.

Received by the editors January 3, 1986.

1980 Mathematics Subject Classification (1985 Revision). Primary 46E25; Secondary 30D60.

Key words and phrases. Symbolic calculus, inverse-closed algebra, Carleman classes.

Research supported by grants of NSERC of Canada and FCAR of Quebec. 
Given a sequence $M=\left\{M_{n}\right\}$ of positive numbers, we define the sequence $M^{\alpha}=\left\{M_{n}^{\alpha}\right\}$ by the rule: $N_{n}=n^{(1-\alpha) n} M_{n}, N_{n}^{c}=n^{(1-\alpha) n} M_{n}^{\alpha}$, where $\left\{\log N_{n}^{c}\right\}$ is the largest convex minorant of the sequence $\left\{\log N_{n}\right\}$ (cf. S. Mandelbrojt [5]). The class $\mathscr{C}_{M}\left(I_{\alpha}\right)$ coincides with the class $\mathscr{C}_{M^{\alpha}}\left(I_{\alpha}\right)$ (cf. for example J. A. Siddiqi and A. El Koutri [7, Theorem 1]). Using Leibnitz' formula and the fact that $\left\{\log N_{n}^{c}\right\}$ is convex, it can be easily seen that $\mathscr{C}_{M^{\alpha}}\left(I_{\alpha}\right)$ is an algebra.

Our main result is as follows:

THEOREM 1. The following assertions are equivalent:

(a) The sequence $A^{\alpha}=\left\{A_{n}^{\alpha}\right\}$, where $A_{n}^{\alpha}=\left(M_{n}^{\alpha} / n !\right)^{1 / n}$, is almost increasing.

(b) If $f \in \mathscr{C}_{M}\left(I_{\alpha}\right)$ and $g$ is analytic in a domain containing the range of $f$, then $g \circ f \in \mathscr{C}_{M}\left(I_{\alpha}\right)$.

(c) $\mathscr{C}_{M}\left(I_{\alpha}\right)$ is inverse-closed.

Proof. That (a) implies (b) follows directly from the formula of Faà di Bruno, viz.,

$$
\begin{array}{r}
(g \circ f)^{(n)}(z)=\sum_{\begin{array}{c}
k_{1}+k_{2}+\cdots+k_{n}=k \\
k_{1}+2 k_{2}+\cdots+n k_{n}=n
\end{array}} \frac{n !}{k_{1} ! k_{2} ! \cdots k_{n} !} g^{(k)}(f(z))\left(\frac{f^{\prime}(z)}{1 !}\right)^{k_{1}} \\
\cdots\left(\frac{f^{(n)}(z)}{n !}\right)^{k_{n}} .
\end{array}
$$

Trivially (b) implies (c). We now show that (c) implies (a). Put $N_{n}^{c}=n^{(1-\alpha) n} M_{n}^{\alpha}$. The sequence $\left\{N_{n}^{c}\right\}$ is log-convex in view of the regularization procedure adopted for obtaining $\left\{M_{n}^{\alpha}\right\}$. Let

$$
f(z)=\sum_{k=0}^{\infty} \frac{1}{2^{k}} N_{k}^{c}\left(\frac{N_{k+1}^{c}}{N_{k}^{c}}\right)^{-k} h\left(\frac{N_{k+1}^{c}}{N_{k}^{c}} z\right),
$$

where

$$
h(z)=\sum_{\nu=0} \frac{(-z)^{\nu}}{[(\nu+1)(2-\alpha)+1] !} \quad(x !=\Gamma(x+1))
$$

Since

$$
\left|h^{(n)}(z)\right| \leqslant 2 \frac{n ! e^{n}}{n^{(2-\alpha) n}}
$$

and

$$
\left(N_{k+1}^{c} / N_{k}^{c}\right)^{n-k} \leqslant N_{n}^{c} / N_{k}^{c}
$$

for all $k$, because of the fact that $\left\{N_{n}^{c}\right\}$ is log-convex, we have

$$
\left|f^{(n)}(z)\right| \leqslant \sum_{k=0}^{\infty} \frac{1}{2^{k}} N_{k}^{c}\left(\frac{N_{k+1}^{c}}{N_{k}^{c}}\right)^{n-k}\left|h^{(n)}\left(\frac{N_{k+1}^{c}}{N_{k}^{c}} z\right)\right| \leqslant 4 e^{n} M_{n}^{\alpha}
$$


for all $z \in I_{\alpha}$. Moreover

$$
\begin{aligned}
f^{(n)}(0) & =\left\{\sum_{k=0}^{\infty} \frac{1}{2^{k}} N_{k}^{c}\left(\frac{N_{k+1}^{c}}{N_{k}^{c}}\right)^{n-k}\right\} \frac{n !(-1)^{n}}{[(n+1)(2-\alpha)+1] !} \\
& =t_{n} \frac{n !(-1)^{n}}{[(n+1)(2-\alpha)+1] !},
\end{aligned}
$$

where $t_{n} \geqslant N_{n}^{c} / 2^{n}$.

Let $g(z)=1 /(\lambda-z)$, where $\lambda>M_{0}^{\alpha}$. Since $\lambda-f \in \mathscr{C}_{M^{\alpha}}\left(I_{\alpha}\right)$ and $\mathscr{C}_{M^{\alpha}}\left(I_{\alpha}\right)$ is inverse-closed, it follows that

$$
g \circ f=(\lambda-f)^{-1} \in \mathscr{C}_{M^{\alpha}}(I) .
$$

We, therefore, have

$$
\begin{aligned}
& \left|(g \circ f)^{(n)}(0)\right| \\
& \quad=\mid \begin{array}{c}
\sum_{\substack{k_{1}+k_{2}+\cdots+k_{n}=k \\
k_{1}+2 k_{2}+\cdots+n k_{n}=n}} \frac{n !}{k_{1} ! \cdots k_{n} !} \frac{k !}{(\lambda-f(0))^{k+1}}\left(\frac{f^{\prime}(0)}{1 !}\right)^{k_{1}} \cdots\left(\frac{f^{(n)}(0)}{n !}\right)^{k_{n}} \mid \\
\leqslant C \lambda^{n} M_{n}^{\alpha}
\end{array}
\end{aligned}
$$

so that

$$
\begin{aligned}
\sum_{\substack{k_{1}+k_{2}+\cdots+k_{n}=k \\
k_{1}+2 k_{2}+\cdots+n k_{n}=n}} \frac{n !}{k_{1} ! \cdots k_{n} !} \frac{k !}{(\lambda-f(0))^{k+1}}\left(\frac{t_{1}}{(2 \gamma+1) !}\right)^{k_{1}} \\
\\
\cdots\left(\frac{t_{n}}{((n+1) \gamma+1) !}\right)^{k_{n}} \leqslant C \lambda^{n} M_{n}^{\alpha},
\end{aligned}
$$

where $\gamma=1-\alpha$.

If we choose $k_{l}=k, l k=n, k_{1}=\cdots=k_{l-1}=k_{l+1}=\cdots=k_{n}=0$, then we have

$$
\left(\frac{t_{l}}{((l+1) \gamma+1) !}\right)^{k} \leqslant C_{1} \lambda_{1}^{n} \frac{M_{n}^{\alpha}}{n !}
$$

or

$$
\left(\frac{M_{l}^{\alpha}}{l !}\right)^{1 / l} \leqslant K_{1}\left(\frac{M_{n}^{\alpha}}{n !}\right)^{1 / n}
$$

If $n$ is not a multiple of $l$, let $l m \leqslant n \leqslant l(m+1)$ so that, using Stirling formula and the fact that $\left\{\left(N_{n}^{c}\right)^{1 / n}\right\}$ is increasing, we get

$$
\left(\frac{M_{n}^{\alpha}}{n !}\right)^{1 / n}=\frac{\left(n^{(1-\alpha) n} M_{n}^{\alpha}\right)^{1 / n}}{(n !)^{1 / n} n^{1-\alpha}} \geqslant \frac{\left((m l)^{(1-\alpha) m l} M_{m l}^{\alpha}\right)^{1 / m l}}{(n !)^{1 / n} n^{1-\alpha}} \geqslant \frac{1}{2^{2-\alpha} K_{1} e} \cdot\left(\frac{M_{l}^{\alpha}}{l !}\right)^{1 / l} .
$$

Thus for $n \geqslant l$

$$
\left(\frac{M_{l}^{\alpha}}{l !}\right)^{1 / l} \leqslant K\left(\frac{M_{n}^{\alpha}}{n !}\right)^{1 / n}
$$

where $K$ is independent of $l$ and $n$ and this completes the proof of the theorem. 
If $\alpha=0$, then in the notation of S. Mandelbrojt (cf. [5, p. 227]) $M_{n}^{0}=M_{n}^{d}$ for all $n \geqslant 1$ and Theorem 1 yields the following characterization of the inverse-closed algebra $\mathscr{C}_{M}\left(\mathbf{R}_{+}\right)$.

THEOREM 2. The following assertions are equivalent:

(a) The sequence $A^{d}=\left\{A_{n}^{d}\right\}$, where $A_{n}^{d}=\left(M_{n}^{d} / n !\right)$, is almost increasing.

(b) If $f \in \mathscr{C}_{M}\left(\mathbf{R}_{+}\right)$and $g$ is analytic in a domain containing the range of $f$, then $g \circ f \in \mathscr{C}_{M}\left(\mathbf{R}_{+}\right)$.

(c) $\mathscr{C}_{M}\left(\mathbf{R}_{+}\right)$is inverse-closed.

\section{REFERENCES}

1. J. Boman and L. Hörmander, Classes of infinitely differentiable functions (mimeographed notes), Stockholm, 1962.

2. J. Bruna, On inverse closed algebras of infinitely differentiable functions, Studia Math. 69 (1980), 61-67.

3. B. I. Korenbljum, Conditions of non-triviality of certain classes of functions analytic in a sector and problems of quasi-analyticity, Soviet Math. Dokl. 7 (1966), 232-236.

4. P. Malliavin, Calcul symbolique et sous algèbres de $L^{1}(G)$, Bull. Soc. Math. France 87 (1959), 181-186.

5. S. Mandelbrojt, Séries adhérentes, regulatisation des suites, applications, Gauthier-Villars, Paris, 1952.

6. W. Rudin, Division in algebras of infinitely differentiable functions, J. Math. Mech. 2 (1962), 797-809.

7. J. A. Siddiqi and A. El Koutri, Cartan-Gorny-Kolmogorov type inequalities for generators of analytic semi-groups and cosine operator functions, preprint.

Departement de Mathematiques, Statistique et Actuariat, Université laval, Cite UniVERSITAIRE, QUEBEC, G1K 7P4, CANADA 